\title{
Aldolase B Overexpression is Associated with Poor Prognosis and Promotes Tumor Progression by Epithelial-Mesenchymal Transition in Colorectal Adenocarcinoma
}

\author{
Qingguo Lia $i^{a, b}$ Yaqi Lia,b Junyan X ${ }^{b, c}$ Sheng Wanga,b Ye Xu ${ }^{a, b} \quad$ Xinxiang Lia,b \\ Sanjun Cai ${ }^{a, b}$
}

aDepartment of Colorectal Surgery, Fudan University Shanghai Cancer Center, Shanghai, 'D Department of Oncology, Shanghai Medical College, Fudan University, Shanghai, 'Department of Nuclear Medicine, Fudan University Shanghai Cancer Center, Shanghai, China

\section{Key Words}

Colorectal cancer • Aldolase B • Epithelial-mesenchymal transition

\begin{abstract}
Background: Glycolysis is considered to be the root of cancer development and progression, which involved a multi-step enzymatic reaction. Our study aimed at figuring out which glycolysis enzyme participates in the development of colorectal cancer and its possible mechanisms. Methods: We firstly screened out Aldolase B (ALDOB) by performing qRT-PCR arrays of glycolysis-related genes in five paired liver metastasis and primary colorectal tissues, and further detected ALDOB protein with immunohistochemistry in tissue microarray (TMA) consisting of 229 samples from stage I-III colorectal cancer patients. CRISPR-Cas9 method was adopted to create knock out colon cancer cell lines (LoVo and SW480) of ALDOB. The effect of ALDOB on cell proliferation and metastasis was examined in vitro using colony formation assay as well as transwell migration and invasion assay, respectively. Results: In TMA, there was $64.6 \%$ of samples demonstrated strong intensity of ALDOB. High ALDOB expression were associated with poor overall survival and disease-free survival in both univariate and multivariate regression analyses $(P<0.05)$. In vitro functional studies of CCK-8 demonstrated that silencing ALDOB expression significantly $(P<0.05)$ inhibited proliferation, migration and invasion of colon cancer cells. Mechanically, silencing ALDOB activated epithelial markers and repressed mesenchymal markers, indicating inactivation of ALDOB may lead to inhibition of epithelial-mesenchymal transition (EMT). Conclusion: Upregulation of ALDOB promotes colorectal cancer metastasis by facilitating EMT and acts as a potential prognostic factor and therapeutic target in colorectal cancer.
\end{abstract}

Q. Li and Y. Li contributed equally to this work.

Sanjun Cai, MD, Ph D.

or Xinxiang Li, MD, Ph D.

KARGER
Department of Colorectal Surgery, Fudan University Shanghai Cancer Center Department of Oncology, Shanghai Medical College, Fudan University 270 Dong'an Road, Xuhui District,Shanghai, 20032 (China)

Tel. +86-021-64175590, E-Mail caisanjun_sh@163.com / Ixx1149@163.com 


\section{Introduction}

Colorectal cancer (CRC) is the third most commonly diagnosed cancer and the third leading cause of cancer deaths in the US [1]. In China, the incidence of CRC has been increasing in recent years as living conditions improve and eating habits change. Surgical resection remains the only curative treatment option for CRC [2]. However, a considerable proportion of CRC patients develop recurrence or metastasis within 5 years after surgical treatment. Thus, a better understanding of the underlying mechanisms that promote the pathogenesis of CRC is urgently needed.

Unlike many differentiated cells in an adult organism, tumor cells have an unrestricted capacity to divide and proliferate. Relentless biosynthesis of macromolecules that are needed for the growth of newly divided cells requires the uptake of glucose and other carbon sources in excess of energetic needs $[3,4]$. The vast majority of tumor cell types display modified rates and pathways of energetic and anabolic metabolism in comparison to their tissue of origin [5]. The Warburg effect, also known as aerobic glycolysis, is a shift from oxidative phosphorylation to glycolysis, a feature of which is increased lactate production even at normal oxygen concentrations, and is considered to be the root of cancer development and progression $[6,7]$.

In the present study, we performed qRT-PCR arrays of glycolysis-related genes in five paired liver metastasis tissues and primary colorectal tissues. Aldolase B (ALDOB) was found mostly overexpressed in metastasis tissues and chosen for further research. Thus, we then focused on investigation of ALDOB expression in CRC tissues and its clinical significance and biological function in colon cancer cells, and we also explored the underlining mechanisms regulated by ALDOB in colon cancer cells.

\section{Material and Methods}

\section{Patients and samples}

To detect the clinical importance of ALDOB expression in cases with CRC, a tissue microarray (TMA) consisted of 229 patients with pathologically and clinically confirmed CRC was used for IHC study, including 19 cases at stage I, 68 at stage II, 106 at stage III, and 36 at stage IV. All patients included underwent primary tumor resection. The characteristics of the samples are shown in Table 1. Construction of the TMA was performed as described previously [8].

RNA extraction, reverse transcription, and qRT-PCR analysis

Total RNA was isolated from five paired tissues from patients with synchronous liver metastases using TRIzol ${ }^{\circledR}$ reagent (15596-026, Invitrogen). A PrimeScript ${ }^{\mathrm{TM}}$ RT Master Mix (Perfect Real Time) kit (RR036A, Takara) was used to synthesize first-strand cDNA from total RNA. After that, SYBR Green realtime PCR assays were performed using ABI 7900HT (Applied Biosystems, USA). The expression level of RNA was normalized to the level of $\beta$-actin. The primers for qRT-PCR analysis were synthesized by Huagene (Shanghai, PRC), the sequences of which are shown in Table 1.

\section{Immunohistochemical staining staining}

Immunohistochemical staining (IHC) staining was performed as described previously [8]. Commercial monoclonal antibodies to ALDOB (titer 1:100, ab133333, Abcam), which has weak cross react with ALDOC (according to data provided by the manufacturer), was used and PBS was used as a negative control. No positive control has been done due to lack of liver tissue sections. Evaluation of the result was performed according the criteria as recommended by the manufacturer. The staining intensity was scored as 0 (negative), 1 (weak), 2 (medium) or 3 (strong). Extent of staining was scored as $0(<5 \%), 1(5-25 \%), 2$ (26$50 \%), 3(51-75 \%)$ and $4(>75 \%)$ according to the percentages of the positive staining areas in relation to the whole carcinoma area. Scores for staining intensity and percentage of positive cells were then multiplied to generate the immunoreactivity score (IRS) for each case. Samples having a final staining score of $\leq 4$ were considered to be low and those with score of $>4$ were considered to be high. 


\section{Cellular Physiology Cell Physiol Biochem 2017;42:397-406 \begin{tabular}{l|l|l} 
and Biochemistry 10.1159/000477484 & $\begin{array}{l}\text { ( ) 2017 The Author(s). Published by S. Karger AG, Basel } \\
\text { www.karger.com/cpb }\end{array}$
\end{tabular} \\ Li et al.: ALDOB in Colorectal Cancer}

\section{CRISPR/CAS9 knockout}

Colon cancer cells (LoVo, SW480) were transfected with lentiCas9-Blast (Addgene \#52962) virus and selected by blasticidin for 5 days. The stable blasticidin resistant sub-lines were then infected with ALDOB specific lenti-gRNA-puro (Addgene \#52963) lentivirus. The guide sequence was preselected from three different guideRNA pairs using in silico on- and off-target predictions (http:// crispr.mit.edu/). The sgRNA target sequences for ALDOB were 5'- ATTACCTCTGGTTCAACAAT-3' (sgRNA 1\#), 5'- AATATCCTTACCTTGAATGG-3' (sgRNA 2\#). The sgRNA sequence targeting firefly GFP was used as control, which was 5'GCACTACCAGAGCTAACTCA-3'. Stably transfected cells were isolated using puromycin selection after the cells were transfected with expression vector or control plasmids. The knockout effect was tested by western blot of proteins extracted in a population of knockout LoVo and SW480 cells.

\section{Western blot}

Briefly, equal quantities of cellular proteins were resolved by sodium dodecyl sulfatepolyacrylamide gel electrophoresis, transferred onto polyvinylidene difluoride membranes, and immunoblotted with primary antibody for detection of ALDOB(titer 1:1,000, ab133333, Abcam), E-cadherin (titer 1/1,000 dilution; Abcam), $\mathrm{N}$-cadherin (titer 1/10,000 dilution; Abcam), and Vimentin (titer 1/1,000 dilution; Abcam) overnight at $4^{\circ} \mathrm{C}$. After incubation with a secondary antibody, blots were visualized using ECL (Pierce, Thermo Scientific, USA) and detected using a BioImaging System. Relative protein expression was normalized to $\beta$-actin.
Table 1 Primers sequences used in the text

\begin{tabular}{|c|c|}
\hline Gene & Primers \\
\hline GLUT1 & $\begin{array}{l}\text { Forward: CTTTGTGGCCTTCTTTGAAGT } \\
\text { Reverse: CCACACAGTTGCTCCACAT }\end{array}$ \\
\hline HK2 & $\begin{array}{l}\text { Forward: GATTGTCCGTAACATTCTCATCGA } \\
\text { Reverse: TGTCTTGAGCCGCTCTGAGAT }\end{array}$ \\
\hline GLUT4 & $\begin{array}{l}\text { Forward: TGGAAGGAAAAGGGCCATGCTG } \\
\text { Reverse: CAATGAGGAATCGTCCAAGGATG }\end{array}$ \\
\hline G6P & $\begin{array}{l}\text { Forward: GGTACACAGGCAAGACCATC } \\
\text { Reverse: GTTTTGGCAATGTGAGTTCC }\end{array}$ \\
\hline PFKM & $\begin{array}{l}\text { Forward: GATACTATGGATGATCCAGACAC } \\
\text { Reverse: GACAGCAATGGCTTTGCCAATC }\end{array}$ \\
\hline PFKL & $\begin{array}{l}\text { Forward: CACAGGTGCCAACATCTTCCGCA } \\
\text { Reverse: TCATGTCGGTGCCGCAGAAGTCG }\end{array}$ \\
\hline PFKP & $\begin{array}{l}\text { Forward: AGAGGACCTTCGTTCTGGAGGT } \\
\text { Reverse: GGGCACGGTTCTCCGAGAGTTT }\end{array}$ \\
\hline ALDOA & $\begin{array}{l}\text { Forward: GTTATCAAATCCAAGGGCGGTGTT } \\
\text { Reverse: AGTCAGCTCCGTCCTTCTTGTAC }\end{array}$ \\
\hline ALDOB & $\begin{array}{l}\text { Forward: CACCATTCAAGGGCTTGATGGCCT } \\
\text { Reverse: TTCCTGGATAGCGAGGCTGGAT }\end{array}$ \\
\hline GAPDH & $\begin{array}{l}\text { Forward: GCACCGTCAAGGCTGAGAAC } \\
\text { Reverse: TGGTGAAGACGCCAGTGGA }\end{array}$ \\
\hline PGK1 & $\begin{array}{l}\text { Forward: CAAGGTTAAAGCCGAGCCAGCCAA } \\
\text { Reverse: GCCTTCTGTGGCAGATTGACTCC }\end{array}$ \\
\hline PGAM1 & $\begin{array}{l}\text { Forward: ATGATGTCCCACCACCTCCGAT } \\
\text { Reverse: ATCCTTCAGACTCTCACAGGAG }\end{array}$ \\
\hline ENO1 & $\begin{array}{l}\text { Forward: GCTCCGGGACAATGATAAGACTCG } \\
\text { Reverse: CTGTTCCATCCATCTCGATCATC }\end{array}$ \\
\hline LDHA & $\begin{array}{l}\text { Forward: TGGAGATTCCAGTGTGCCTGTATGG } \\
\text { Reverse: CACCTCATAAGCACTCTCAACCACC }\end{array}$ \\
\hline LDHB & $\begin{array}{l}\text { Forward: GGAAGGAAGTGCATAAGATGGTGG } \\
\text { Reverse: CCCCTTTACCATTGTTGACACG }\end{array}$ \\
\hline PKM1 & $\begin{array}{l}\text { Forward: CTATCCTCTGGAGGCTGTGC } \\
\text { Reverse: CCATGAGGTCTGTGGAGTGA }\end{array}$ \\
\hline PKM2 & $\begin{array}{l}\text { Forward: CAAAGGACCTCAGCAGCCATGTC } \\
\text { Reverse: GGGAAGCTGGGCCAATGGTACAGA }\end{array}$ \\
\hline FBP1 & $\begin{array}{l}\text { Forward: CCCCAGATAATTCAGCTCCTTA } \\
\text { Reverse: GTTGCATTCGTACAGCAGTCTC }\end{array}$ \\
\hline FBP2 & $\begin{array}{l}\text { Forward: TCAACATGGTCCAATCCTCCT } \\
\text { Reverse: CATCCAGTGGGTCAAAGCAGA }\end{array}$ \\
\hline$\beta$-actin & $\begin{array}{l}\text { Forward: CTACGTCGCCCTGGACTTCGAGC } \\
\text { Reverse: GATGGAGCCGCCGATCCACACGG }\end{array}$ \\
\hline
\end{tabular}

\section{CCK8 assay}

Cells transfected with ALDOB-sgRNA or GFP-sgRNA lentivirus were plated in 96-well plates at density of $2 \times 10^{3}$ cells/well and cultured for $24,48,72$ or $96 \mathrm{~h}$, respectively. $10 \mu \mathrm{L}$ of CCK8 solution (Dojindo Laboratories, Kumamoto, Japan) was added to each well, incubated for $2 \mathrm{~h}$ at $37^{\circ} \mathrm{C}$ and read at $450 \mathrm{~nm}$. All experiments were performed 3 times.

\section{Colony formation assay}

Cells infected with ALDOB-sgRNA lentivirus or GFP-sgRNA lentivirus was seeded in 6-well plates at a density of 400 per well and cultured at $37^{\circ} \mathrm{C}$ for 14 days. Cells were washed twice with PBS, fixed with $4 \%$ paraformaldehyde, stained with Giemsa for 10 minutes and washed 3 times with double distilled $\mathrm{H}_{2} \mathrm{O}$. The colonies containing more than 50 cells were counted using light microscopy. Colony-forming efficiency (CFE $\%$ ) was defined as the ratio of the number of colonies formed in culture to the number of cells inoculated.

\section{In vitro cell migration and invasion assays}

The migration and invasion assays were conducted in a 24-well Transwell chamber (Costar, Cambridge, MA) with uncoated membranes or membranes coated with Matrigel (BD Biosciences, San Jose, CA, USA). Cells $\left(4 \times 10^{4}\right.$ per well $)$ were resuspended in serum-free medium and seeded into the upper chamber. Culture medium containing $10 \%$ fetal bovine serum was added to the lower chamber as the chemoattractant. The 


\section{Cellular Physiology Cell Physiol Biochem 2017;42:397-406

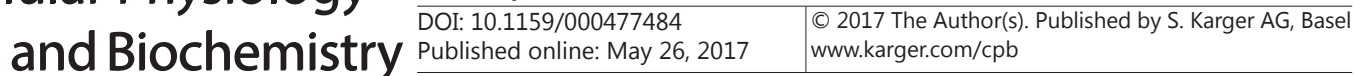 \\ Li et al.: ALDOB in Colorectal Cancer}

cells were incubated in a humidified incubator at $37^{\circ} \mathrm{C}$ for 48 hours (migration assays) or 72 hours (invasion assays). Non-invading cells in the upper chambers were removed with cotton swabs. The cells attached to the lower surface were fixed and stained with crystal violet. The number of cells which were attached to the lower surface was counted in five random fields under a microscope $(\times 200)$.

\section{Ethic statement}

This study was approved by the Shanghai Cancer Center Research Ethics Committee. The methods were carried out in accordance with the approved guidelines. Informed consent was obtained from each patient according to the committee's regulations.

\section{Statistical Analysis}

The statistical associations between clinical parameters and IHC staining were tested by $\chi^{2}$ test to compare the expected frequencies and the observed frequencies in two groups. The 5-year overall survival (OS) and disease-free survival (DFS) were calculated by the Kaplan-Meier method to estimate the survival and hazard functions of censored data. The difference in survival between the groups at each observed event time was compared by the log rank test. Variables that seemed to be significantly associated with survival in univariate analysis were included into multivariate analysis, which was performed with Cox proportional hazard model to relate survival to a collection of other risk factors. A $P$ value of $<0.05$ was considered statistically significant. All statistical analyses were carried out by SPSS for Windows, version 21.0 (SPSS, Chicago, IL).

\section{Results}

Glycolytic enzymes ALDOB might promote tumor metastasis in colorectal cancer

Glycolysis is a multi-step enzymatic reaction involving with a series of rate-limiting enzymes (Fig.1A). Recent studies have provided evidence that some glycolytic enzymes are more complicated, multifaceted proteins rather than simple components of the glycolytic pathway [9]. In order to find the potential enzymes that may be involved in cancer metastasis, qRT-PCR assay was used to measure the levels of glycolytic enzyme mRNA expression in five paired tissues from patients with synchronous liver metastasis, who all underwent synchronous primary tumor and liver metastases resection. We found that the expression of ALDOB changed most significantly in liver metastases compared with corresponding primary tumors (Fig.1B).

Fig. 1. (A) Glycolysis is a multi-step enzymatic reaction involving with a series of rate-limiting enzymes. (B) Heatmap of differentially expressed genes in five paired primary colorectal cancer tissues and liver metastases using qRT-PCR and the results were normalized by Z-score. Each column represents a specimen which is denoted on the above and each role represents a gene which is denoted on the right. Red color indicates genes that were upregulated and green color indicates genes that were downregulated. P: primary colorectal cancer tissue; M: corresponding liver metastasis.

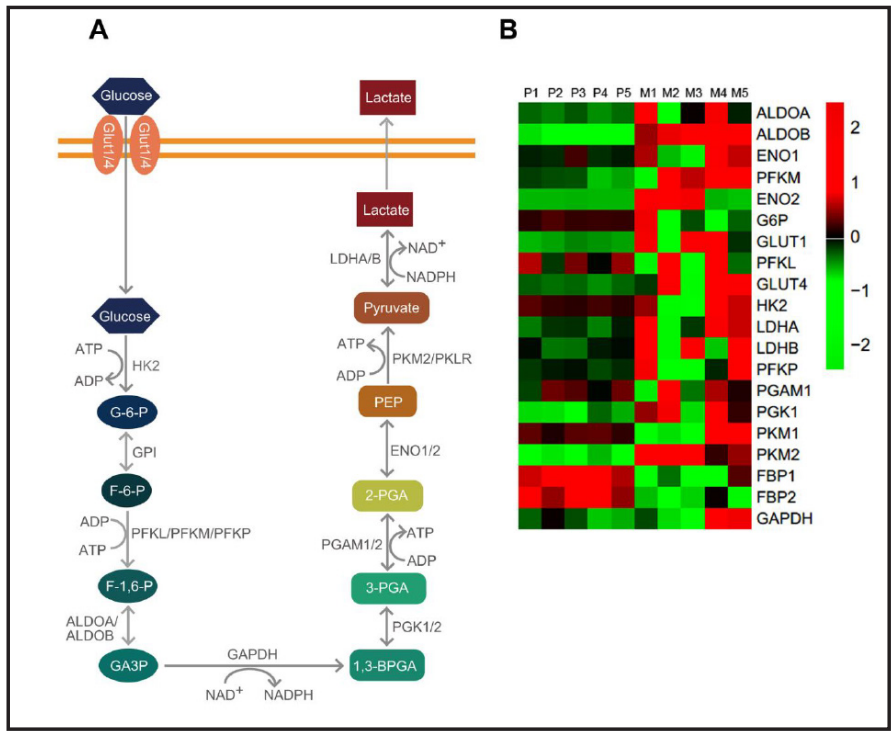


Fig. 2. Overexpression of ALDOB is correlated with survival of colorectal patients. ALDOB expression was detected by immunohistochemical staining in TMA. ALDOB was mainly localized with the cytoplasmic of cancer cell. (A) Example of low ALDOB expression in normal colorectal tissues. (B) Example of low ALDOB expression in primary colorectal cancer at stage II. (C) Example of high ALDOB expression in primary colorectal cancer at stage III. (D) Negative control. As visualized in a 200×and $400 \times$ magnifications (lower panels). Patients included in TMA were then divided into ALDOB-high group ( $\mathrm{N}=148)$ and ALDOB-low group $(\mathrm{N}=81)$ and Kaplan-Meier analysis was performed to evaluate the correlation of ALDOB expression and patients survival. (E) Overexpression of ALDOB was correlated with worse OS $\left(\chi^{2}=16.160, P<0.001\right)$. (F) Overexpression of ALDOB was correlated with worse DFS $\left(\chi^{2}=\right.$ 21.872, $P<0.001$ ).

\section{ALDOB overexpression was} associated with worse clinical outcome in CRC

To explore the clinical significance of ALDOB overexpression, IHC was used to detect ALDOB protein expression in a TMA containing 229 CRC samples. ALDOB was positively stained in the cytoplasm of the cancer cells. Analysis of protein expressions in TMA revealed that $64.6 \%(148 / 229)$ of samples demonstrated strong $(2+$ and $3+$ ) intensity of ALDOB. IHC staining of ALDOB are illustrated in Fig. 2A-D.

According to IRS of each patients, $64.6 \%(148 / 229)$ of patients were assigned to the ALDOB-high group and $35.4 \%(81 / 229)$ of patients to the ALDOB-low group. The correlation of ALDOB in CRC with clinical features is provided in Table 2. The positive expression of ALDOB was noted more often in patients with more advanced tumor stage $(P<0.05)$.

At last follow-up, 71 patients died and 87 patients experienced tumor recurrence. The $\mathrm{OS}$ rates at 3 and 5
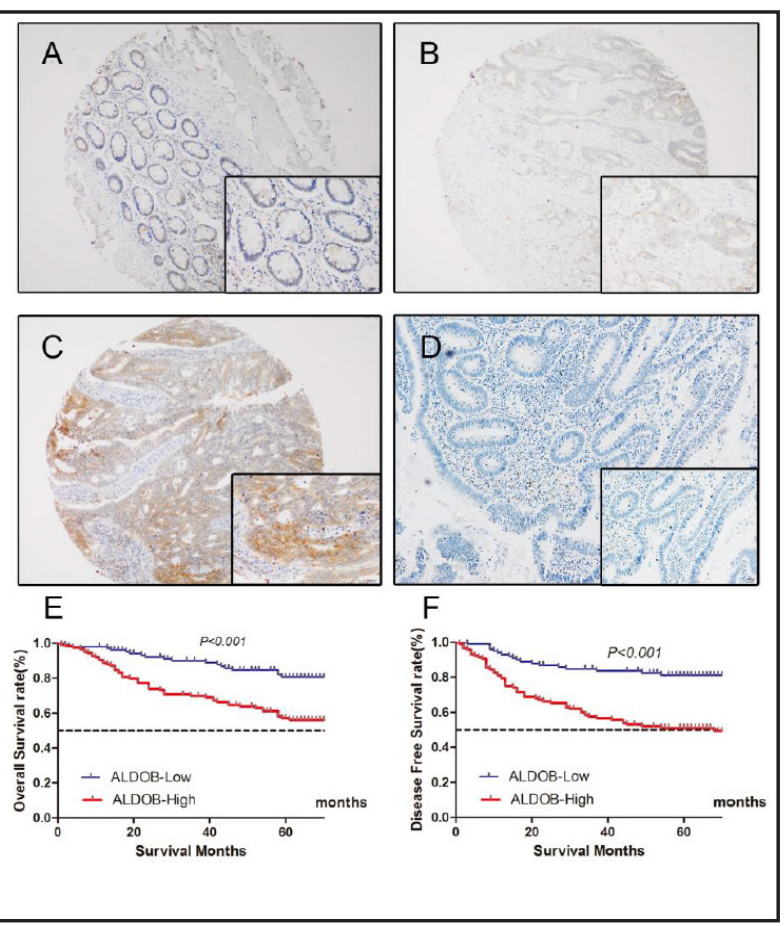

Table 2 Comparison of baseline clinical characteristics based on ALDOB expression level

\begin{tabular}{|c|c|c|c|}
\hline Variable & & $\begin{array}{c}\text { ALDOB } \\
\text { Low }(\%) \text { High (\%) }\end{array}$ & $P$ \\
\hline Sex & & & 0.292 \\
\hline & Male & $44(54.3) 91(61.5)$ & \\
\hline & Female & $37(45.7) 57(38.5)$ & \\
\hline Age & & & 0.053 \\
\hline & $\geq 60$ & $56(69.1) 83(56.1)$ & \\
\hline & $<60$ & $25(30.9) 65(43.9)$ & \\
\hline Primary site & & & 0.150 \\
\hline & Colon & $33(40.7) 75(50.7)$ & \\
\hline & Rectum & $48(59.3) 73(49.3)$ & \\
\hline Histotype & & & 0.488 \\
\hline & Adenocarcinoma & $78(96.3) 136(91.9)$ & \\
\hline & $\begin{array}{l}\text { Mucinous/signet ring } \\
\text { cell }\end{array}$ & $3(3.7) \quad 12(8.1)$ & \\
\hline CEA(ug/ml) & & & $0.127^{*}$ \\
\hline & $<5.0$ & $57(70.4) 84(56.8)$ & \\
\hline & $\geq 5.0$ & $22(27.2) 58(39.2)$ & \\
\hline & Unknown & $2(2.5) \quad 6(4.1)$ & \\
\hline LNs retrieval & & & 0.697 \\
\hline & $<12$ & $10(12.3) 21(14.2)$ & \\
\hline & $\geq 12$ & $71(87.7) 127(85.8)$ & \\
\hline AJCC Stage & & & 0.001 \\
\hline & I & $11(13.6) 8(5.4)$ & \\
\hline & II & $30(37.0) 38(25.7)$ & \\
\hline & III & $36(44.4) 70(47.3)$ & \\
\hline & IV & $4(4.9) \quad 32(21.6)$ & \\
\hline Lymphovascular invasion & & & 0.060 \\
\hline & Negative & $56(69.4) 98(66.2)$ & \\
\hline & Positive & $25(30.9) 50(33.8)$ & \\
\hline Perineural invasion & & & 0.924 \\
\hline & Negative & $70(86.4) 125(84.5)$ & \\
\hline & Positive & $11(13.6) 23(15.5)$ & \\
\hline
\end{tabular}

years were $79.0 \%$ and $68.0 \%$, respectively. The probabilities of DFS rates at 3 and 5 years were $66.0 \%$ and $61.0 \%$, respectively.

The Kaplan-Meier plot demonstrated that high expression of ALDOB was associated with both poor OS and DFS (Fig. 2E, 2F). Besides, in univariate Cox proportional hazard 
Table 3. Univariate and multivariate survival analyses of ALDOB expression and overall survival for patients with colorectal cancer

\begin{tabular}{lllll}
\hline Variable & Univariate analysis & & Multivariate analysis & \\
& HR $(95 \% \mathrm{CI})$ & $P$ & HR $(95 \% \mathrm{CI})$ & $P$ \\
\hline Sex & $0.851(0.527-1.375)$ & 0.510 & & $\mathrm{NI}$ \\
Age & $1.290(0.808-2.059)$ & 0.286 & & $\mathrm{NI}$ \\
Primary site & $0.693(0.434-1.106)$ & 0.124 & & $\mathrm{NI}$ \\
Histotype & $0.814(0.292-2.232)$ & 0.689 & & $\mathrm{NI}$ \\
CEA & $1.614(1.028-2.536)$ & 0.038 & $0.854(0.557-1.311)$ & 0.471 \\
LNs retrieval & $0.674(0.369-1.231)$ & 0.199 & & $\mathrm{NI}$ \\
AJCC Stage & $4.735(3.269-6.858)$ & $<0.001$ & $4.660(3.064-7.088)$ & $<0.001$ \\
Lymphovascular invasion & $2.170(1.361-3.459)$ & 0.001 & $1.074(0.656-1.757)$ & 0.777 \\
Perineural invasion & $1.765(1.011-3.080)$ & 0.046 & $1.337(0.758-2.358)$ & 0.316 \\
ALDOB & $2.844(1.668-4.862)$ & $<0.001$ & $2.411(1.360-4.274)$ & 0.003 \\
\hline
\end{tabular}

NI: not included in multivariate survival analysis, ${ }^{*} P$ values refer to the log-rank test of the differences between the two survival curves generated using Kaplan-Meier analysis

Table 4 Univariate and multivariate survival analyses of ALDOB expression and disease free survival for patients with colorectal cancer

\begin{tabular}{|c|c|c|c|c|}
\hline \multirow[t]{2}{*}{ Variable } & \multicolumn{2}{|l|}{ Univariate analysis } & \multicolumn{2}{|l|}{ Multivariate analysis } \\
\hline & HR $(95 \% \mathrm{CI})$ & $P$ & HR $(95 \% \mathrm{CI})$ & $P$ \\
\hline Sex & $0.718(0.461-1.117)$ & 0.142 & & $\mathrm{NI}$ \\
\hline Age & $1.154(0.755-1.766)$ & 0.508 & & NI \\
\hline Primary site & $0.779(0.511-1.186)$ & 0.245 & & NI \\
\hline Histotype & $0.822(0.333-2.029)$ & 0.671 & & NI \\
\hline CEA & $2.013(1.337-3.030)$ & 0.001 & $1.147(0.774-1.701)$ & 0.495 \\
\hline LNs retrieval & $0.676(0.387-1.180)$ & 0.168 & & NI \\
\hline AJCC Stage & $4.958(3.504-7.016)$ & $<0.001$ & $4.904(3.276-7.342)$ & $<0.001$ \\
\hline Lymphovascular invasion & $1.912(1.252-2.920)$ & 0.003 & $0.766(0.477-1.230)$ & 0.270 \\
\hline Perineural invasion & $1.657(0.986-2.785)$ & 0.057 & & NI \\
\hline ALDOB & $2.992(1.844-4.855)$ & $<0.001$ & $2.205(1.309-3.716)$ & 0.003 \\
\hline
\end{tabular}

NI: not included in multivariate survival analysis, * $P$ values refer to the log-rank test of the differences between the two survival curves generated using Kaplan-Meier analysis

analysis, high CEA level, advanced tumor stage, presence of lymphovascular invasion and perineural invasion were significantly associated with poor prognosis in terms of OS $(P<0.05$, Table 3), while high CEA level, advanced tumor stage and presence of perineural invasion were significantly associated with short DFS $(P<0.05$, Table 4). Multivariate Cox proportional hazard analysis after adjustment for all the potential prognostic factors indicated that only tumor stage and ALDOB expression level were the two independent predictors of OS (ALDOB high, HR: 2.411, 95\%CI 1.360-4.274) and DFS (ALDOB high, HR: 2.205, 95\%CI 1.309-3.716) (Table 3, 4).

Altered expression of $A L D O B$ affected migratory and invasive ability of colon cancer cells in vitro

The above results demonstrate that overexpressed ALDOB is correlated with tumor progression and poor survival of colorectal cancer. Thus, we want to explore whether knockdown of ALDOB can inhibit growth of colon cancer cells. Two sgRNAs were designed 
Fig. 3. Knockdown of ALDOB expression suppresses the proliferation and invasion abilities of colon cancer cells. (A) Western blot analysis was performed to examine ALDOB expression in seven colon cancer cell lines. (B) The protein level of ALDOB in LoVo and SW480 cells after transfection of sgRNAs against ALDOB or scramble sequence was analyzed by Western blot. $\beta$-actin was used as the loading control. (C) Growth curves of LoVo and SW480 cells with transfected sgRNAs or scramble sequence. Cell growth was determined by CCK8. (D) A colony formation assay was performed to determine the oncogenic growth of LoVo and SW480 cells transfected with sgRNAs or scramble sequence. (E)Migration and (F) Transwell assays were performed to determine the migratory abilities of sgRNA ALDOB-transfected LoVo and SW480 cells. Data represent the mean \pm SD and are representative of three independent experiments. ${ }^{*} P<0.05$.

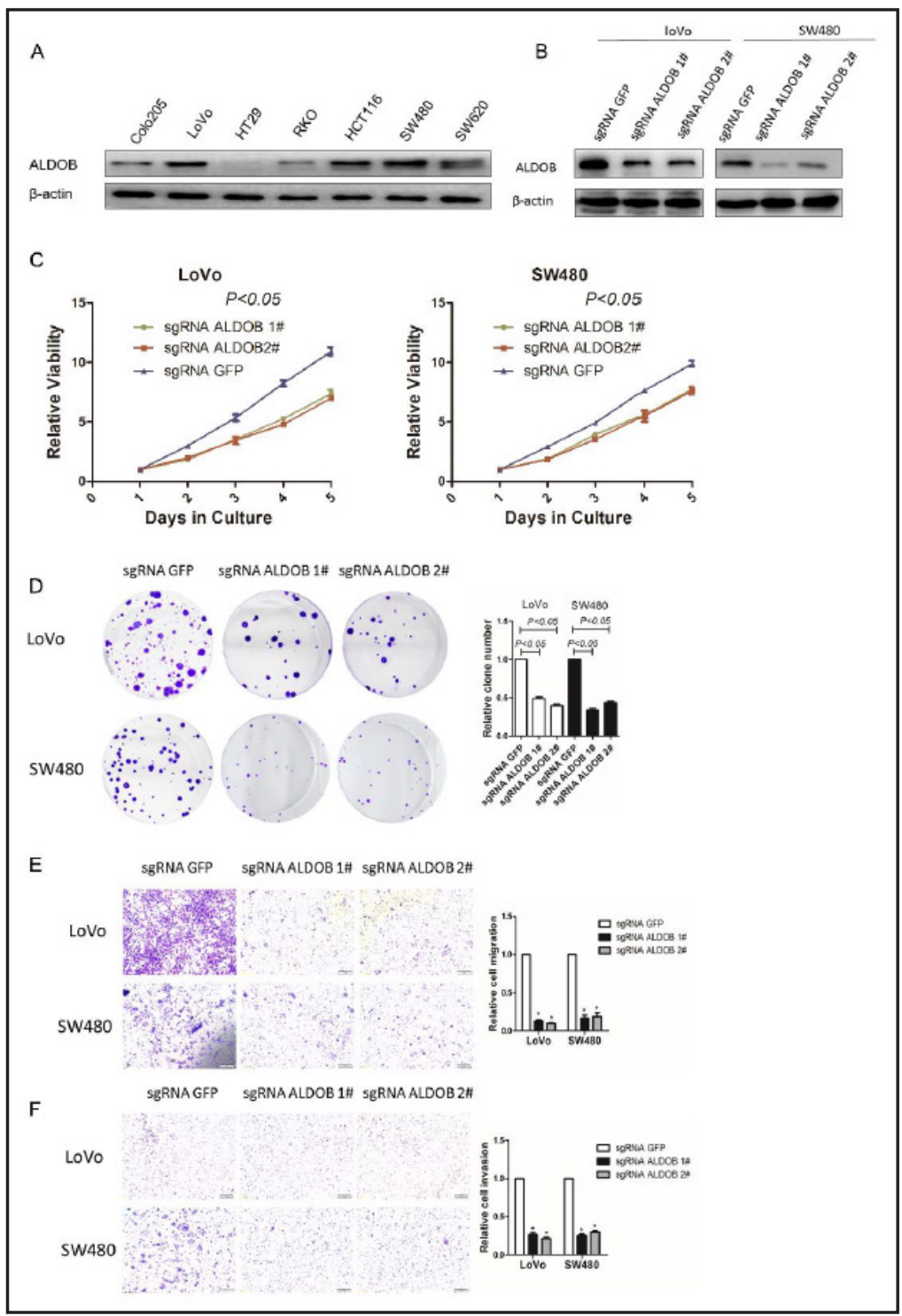

against ALDOB and transfected into two colon cancer cell lines LoVo and SW480, which have high endogenous ALDOB expression (Fig. 3A). Western blot verified that ALDOB protein was inactivated in both LoVo and SW480 cells transfected with the two sgRNAs (Fig. 3B). Cell proliferation assay and colony formation assays showed that proliferation and colony formation abilities of both LoVo and SW480 cells transfected with sgRNA-1 or -2 was remarkably inhibited compared with that of those transfected with control sgRNA (Fig. 3C and 3D), indicating that ALDOB inactivation suppressed cell growth. Transwell migration assay and Matrigel invasion assay showed that silencing ALDOB expression inhibited migration and invasion of LoVo and SW480 cells with statistically significant difference (Fig. 3E and $3 F)(P<0.05)$.These findings suggest that knockdown of ALDOB represses proliferation and invasion ability of colorectal cancer cells.

\section{ALDOB promotes epithelial-mesenchymal transition in human colorectal cancer}

Studies demonstrate that cancer cells may have epithelial-mesenchymal transition (EMT) before metastasis, by which cancer cells lose cell-cell adhesion, apical-basolateral polarity and epithelial markers, and acquire motility, spindle-cell shape and mesenchymal markers [10]. Thus EMT is thought to facilitate cancer cell motility, invasion and metastasis. 


\section{Cellular Physiology Cell Physiol Biochem 2017;42:397-406 \begin{tabular}{l|l} 
DOI: 10.1159/000477484 & a 2017 The Author(s). Published by S. Karger AG, Basel \\
www.karger.com/cpb
\end{tabular}

Fig. 4. ALDOB induces epithelial mesenchymal transition in colon cancer. Western-blot analysis of phenotypic markers including E-cadherin, Vimentin, N-cadherin in LoVo and SW480 cells after transfection of sgRNAs against ALDOB or scramble sequence. $\beta$-actin expression was used as control.

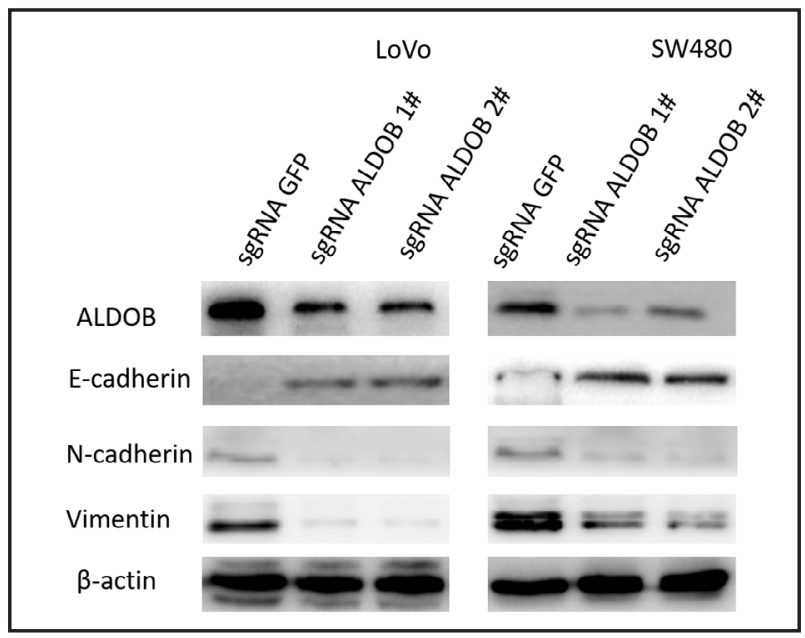

Based on this knowledge and our results above, we hypothesize that ALDOB can induce EMT during its promotion of migration and invasion. To this end, we explored the markers of EMT with Western blot: E-cadherin (epithelial marker), N-cadherin and vimentin (mesenchymal markers). The result showed that silencing ALDOB activated epithelial markers and repressed mesenchymal markers (Fig. 4), indicating inactivation of ALDOB may lead to inhibition of EMT, which needs to be verified by further research.

\section{Discussion}

Aberrant expression and activity of metabolic enzymes are the hallmarks of tumorigenesis and metastasis. Advances in understanding the mechanisms of tumor progression and metastasis have clearly highlighted the importance of the tumor metabolism alteration, which supports not only tumor cells' energy requirements but also their enormous biosynthetic needs. Such metabolic alterations modulate glucose, amino acid, fatty acid-dependent metabolite biosynthesis and energy production [5, 11]. In the 1920s, Warburg made the striking discovery that in contrast to that normal cells produce ATP mainly via mitochondrial oxidative phosphorylation, cancer cells prefer to metabolize glucose via glycolysis, even in the presence of ample oxygen [6]. In the present study, we firstly screened out ALDOB by performing qRT-PCR arrays of glycolysis-related genes in five paired liver metastasis tissues and primary colorectal tissues. We further explored by IHC of TMA and found that the expression levels of glycolytic enzymes ALDOB was significantly higher in cancer tissues than their normal tissues. High ALDOB expression was correlated with advanced tumor stage, and ALDOB expression was an independent prognosis biomarker in CRC. Silencing ALDOB expression inhibited proliferation and invasion in colon cancer cells by inhibiting epithelial-mesenchymal transition (EMT).

It is currently well understood that the Warburg effect plays a critical role in the carcinogenesis and development of cancer. A recent publication has shown that genes involved in aerobic glycolysis are over-expressed in at least 24 different types of cancer corresponding to approximately $70 \%$ of all cancers [12]. Studies have provided evidence that some glycolytic enzymes are more complicated, multifaceted proteins rather than simple components of the glycolytic pathway [9]. These glycolytic enzymes have acquired additional non-glycolytic functions in many aspects. For example, pyruvate kinase M2 (PKM2) facilitates colon cancer cell migration and drug resistance via the modulation of STAT3 signal $[13,14]$. Alpha-enolase (ENO1) promotes cell glycolysis, growth, migration, and invasion in non-small cell lung cancer through FAK-mediated PI3K/AKT pathway [15], and ENO1 is confirmed as a novel substrate of FBXW7 in colorectal cancer [16]. Lactate dehydrogenase A (LDHA) acts as oncogenic role in prostate cancer [17]. Aldolase A (ALDOA) may provide good survival prediction for stage I-IV colorectal cancer patients [18]. GLUT-4 may be up-regulated by p38- 
MAPK signaling to enhance glycolysis in gastric cancer cells [19].

ALDOB, also known as fructose-bisphosphate aldolase, is an important enzyme for glucose and fructose metabolism. It is found primarily in the liver, but it is also present at lower levels in kidney and intestinal cells $[20,21]$. It cleaves fructose1-phosphate to yield glyceraldehyde and dihydroxyacetone phosphate. Accumulation of fructose 1-phosphate in tissues due to a defect in ALDOB may result in hereditary fructose intolerance [22]. Although the role of aldolase in metabolism is well established, there is growing evidence for many alternative functions of this enzyme. In particular, aldolase interacts with various proteins unrelated to glycolytic enzymes, including F-actin [23], ARNO [24], tubulin [25] and is crucial for proliferation [26] and invasion [27] of cancer cells through a non-glycolytic pathway. In the present study, ALDOB was highly expressed in CRC and its expression was correlated with tumor stage. Thus, the expression of ALDOB can be valued as a prognostic biomarker in CRC.

As EMT is one of the key events in tumor invasion and metastasis, and ALDOB expression was correlated with advanced tumor stage, and silencing ALDOB expression inhibited colon cancer cells proliferation and invasion abilities. We then tested whether ALDOB performed its functions on cancer cell by way of inducing EMT pathway. As anticipated, silencing of ALDOB expression led to increased protein levels of epithelial marker (E-cadherin) and decreased expression of mesenchymal marker (N-cadherin, vimentin), suggesting that silencing of ALDOB might lead to a reversion of EMT progression in CRC.

Although the clinical significance of ALDOB in CRC has been revealed, several limitations of this study need further discussion. Firstly, the number of patients enrolled in this study was small, especially for the patients at stage I and stage IV. Secondly, an independent external cohort is necessary to validate our findings. Thirdly, more detailed studies should be conducted to uncover the biological mechanisms of ALDOB in CRC.

In summary, the present study confirmed that ALDOB plays a critical role in CRC by regulating EMT pathway in tumor progression and metastasis and high expression of ALDOB was an independent adverse prognostic factor for both OS and DFS in CRC patients. ALDOB may serves as a new biomarker and potential therapeutic target for CRC treatment.

\section{Acknowledgments}

This research was supported by the National Science Foundation of China (No. 81372646, 81101586), National Key Basic Research Program of China (2014CBA02002) and Shanghai Municipal Natural Science Foundation (17ZR1406400). The funders had no role in the study design, data collection and analysis, decision to publish, or preparation of the manuscript.

\section{Disclosure Statement}

Competing Financial Interests statement: the authors declare no competing financial interests.

\section{Reference}

1 Siegel RL, Miller KD, Jemal A: Cancer statistics, 2015. CA Cancer J Clin 2015;65:5-29.

2 Shin A, Jung KW, Won YJ: Colorectal cancer mortality in Hong Kong of China, Japan, South Korea, and Singapore. World J Gastroenterol 2013;19:979-983.

3 Bauer DE, Harris MH, Plas DR, Lum JJ, Hammerman PS, Rathmell JC, Riley JL, Thompson CB: Cytokine stimulation of aerobic glycolysis in hematopoietic cells exceeds proliferative demand. FASEB J 2004;18:1303-1305.

4 DeBerardinis RJ, Lum JJ, Hatzivassiliou G, Thompson CB: The biology of cancer: metabolic reprogramming fuels cell growth and proliferation. Cell Metab 2008;7:11-20. 


\section{Cellular Physiology Cell Physiol Biochem 2017;42:397-406 \begin{tabular}{l|l} 
DOI: 10.1159/000477484 & $\begin{array}{l}\text { O 2017 The Author(s). Published by S. Karger AG, Basel } \\
\text { www.karger.com/cpb }\end{array}$ \\
\hline
\end{tabular}}

Li et al.: ALDOB in Colorectal Cancer

-5 Moreno-Sanchez R, Rodriguez-Enriquez S, Marin-Hernandez A, Saavedra E: Energy metabolism in tumor cells. FEBS J 2007;274:1393-1418.

6 Warburg 0: On the origin of cancer cells. Science 1956;123:309-314.

7 Garber K: Energy deregulation: licensing tumors to grow. Science 2006;312:1158-1159.

$>8$ Li Q Wu J, Wei P, Xu Y, Zhuo C, Wang Y, Li D, Cai S: Overexpression of forkhead Box C2 promotes tumor metastasis and indicates poor prognosis in colon cancer via regulating epithelial-mesenchymal transition. Am J Cancer Res 2015;5:2022-2034.

-9 Kim JW, Dang CV: Multifaceted roles of glycolytic enzymes. Trends Biochem Sci 2005;30:142-150.

10 Tsuji T, Ibaragi S, Hu GF: Epithelial-mesenchymal transition and cell cooperativity in metastasis. Cancer Res 2009;69:7135-7139.

-11 Shi M, Cui J, Du J, Wei D, Jia Z, Zhang J, Zhu Z, Gao Y, Xie K: A novel KLF4/LDHA signaling pathway regulates aerobic glycolysis in and progression of pancreatic cancer. Clin Cancer Res 2014;20:4370-4380.

12 Altenberg B, Greulich KO: Genes of glycolysis are ubiquitously overexpressed in 24 cancer classes. Genomics 2004;84:1014-1020.

13 Li Q Zhang D, Chen X, He L, Li T, Xu X, Li M: Nuclear PKM2 contributes to gefitinib resistance via upregulation of STAT3 activation in colorectal cancer. Sci Rep 2015;5:16082.

14 Yang P, Li Z, Fu R, Wu H, Li Z: Pyruvate kinase M2 facilitates colon cancer cell migration via the modulation of STAT3 signalling. Cell Signal 2014;26:1853-1862.

15 Fu QF, Liu Y, Fan Y, Hua SN, Qu HY, Dong SW, Li RL, Zhao MY, Zhen Y, Yu XL, Chen YY, Luo RC, Li R, Li LB, Deng XJ, Fang WY, Liu Z, Song X: Alpha-enolase promotes cell glycolysis, growth, migration, and invasion in non-small cell lung cancer through FAK-mediated PI3K/AKT pathway. J Hematol Oncol 2015;8:22.

16 Zhan P, Wang Y, Zhao S, Liu C, Wang Y, Wen M, Mao JH, Wei G, Zhang P: FBXW7 negatively regulates EN01 expression and function in colorectal cancer. Lab Invest 2015;95:995-1004.

-17 Xian ZY, Liu JM, Chen QK, Chen HZ, Ye CJ, Xue J, Yang HQ, Li JL, Liu XF, Kuang SJ: Inhibition of LDHA suppresses tumor progression in prostate cancer. Tumour Biol 2015;36:8093-8100.

18 Peng Y, Li X, Wu M, Yang J, Liu M, Zhang W, Xiang B, Wang X, Li X, Li G, Shen S: New prognosis biomarkers identified by dynamic proteomic analysis of colorectal cancer. Mol Biosyst 2012;8:3077-3088.

19 Liu J, Wen D, Fang X, Wang X, Liu T, Zhu J: p38MAPK Signaling Enhances Glycolysis Through the UpRegulation of the Glucose Transporter GLUT-4 in Gastric Cancer Cells. Cell Physiol Biochem 2015;36:155165.

20 Arakaki TL, Pezza JA, Cronin MA, Hopkins CE, Zimmer DB, Tolan DR, Allen KN: Structure of human brain fructose 1,6-(bis)phosphate aldolase: linking isozyme structure with function. Protein Sci 2004;13:30773084.

21 Tolan DR, Niclas J, Bruce BD, Lebo RV: Evolutionary implications of the human aldolase-A, -B, -C, and -pseudogene chromosome locations. Am J Hum Genet 1987;41:907-924.

22 Ali M, Rellos P, Cox TM: Hereditary fructose intolerance. J Med Genet 1998;35:353-365.

23 Wang J, Morris AJ, Tolan DR, Pagliaro L: The molecular nature of the F-actin binding activity of aldolase revealed with site-directed mutants. J Biol Chem 1996;271:6861-6865.

-24 Merkulova M, Hurtado-Lorenzo A, Hosokawa H, Zhuang Z, Brown D, Ausiello DA, Marshansky V: Aldolase directly interacts with ARNO and modulates cell morphology and acidic vesicle distribution. Am J Physiol Cell Physiol 2011;300:C1442-1455.

25 Volker KW, Knull H: A glycolytic enzyme binding domain on tubulin. Arch Biochem Biophys 1997;338:237243.

26 Ritterson Lew C, Tolan DR: Targeting of several glycolytic enzymes using RNA interference reveals aldolase affects cancer cell proliferation through a non-glycolytic mechanism. J Biol Chem 2012;287:42554-42563.

27 Tao QF, Yuan SX, Yang F, Yang S, Yang Y, Yuan JH, Wang ZG, Xu QG, Lin KY, Cai J, Yu J, Huang WL, Teng XL, Zhou CC, Wang F, Sun SH, Zhou WP: Aldolase B inhibits metastasis through Ten-Eleven Translocation 1 and serves as a prognostic biomarker in hepatocellular carcinoma. Mol Cancer 2015;14:170. 